\title{
An Upper Bound of Large Deviations for Capacities
}

\author{
Xiaomin Cao \\ School of Mathematics, Shandong University, Jinan 250000, China \\ Correspondence should be addressed to Xiaomin Cao; caoxiaomin319320@163.com
}

Received 23 April 2014; Accepted 22 May 2014; Published 15 June 2014

Academic Editor: Xuejun Xie

Copyright (C) 2014 Xiaomin Cao. This is an open access article distributed under the Creative Commons Attribution License, which permits unrestricted use, distribution, and reproduction in any medium, provided the original work is properly cited.

\begin{abstract}
Up to now, most of the academic researches about the large deviation and risk theory are under the framework of the classical linear expectations. But motivated by problems of model uncertainties in statistics, measures of risk, and superhedging in finance, sublinear expectations are extensively studied. In this paper, we obtain a type of large deviation principle under the sublinear expectation. This result is a new expression of the Gärtner-Ellis theorem under the sublinear expectations which is in the classical theory of large deviations. In addition, we introduce a new process under the sublinear expectations, that is, the G-Poisson process. We give an application of our result and obtain the rate function of the compound G-Poisson process in the upper bound of large deviations for capacities. The application of our result opens a new field for the research of risk theory in the future.
\end{abstract}

\section{Introduction}

Large deviation theory is one of the key techniques of modern probability, a role which is emphasized by the recent award of the Abel prize to S.R.S. Varadhan, one of the pioneers of the subject. The large deviation principle characterizes the limiting behavior as $\mathcal{E} \rightarrow \infty$ of a family of probability measures $\mu_{\varepsilon}$ in terms of a rate function. Also Cramér's theorem has been widely known for a long time as a fundamental result in large deviations. It is very useful in many fields. But Cramér's theorem is limited to the i.i.d. case. However, a glance at the proof should be enough to convince the reader that some extension to the non-i.i.d. case is possible. As described in [1], Gärtner-Ellis theorem is a generalization of Cramér's theorem in non-i.i.d situation to conclusions.

Motivated by problems of model uncertainties in statistics, measures of risk, and superhedging in finance, sublinear expectations are extensively studied [2]. Since the paper [3] on coherent risk measures, authors have been more and more interested in sublinear expectations [4, 5]. By Peng [6], we know that a sublinear expectation $\widehat{E}$ can be represented as the upper expectation of a set of linear expectations $\left\{E_{\theta}\right.$ : $\theta \in \Theta\}$; that is, $\widehat{E}[\cdot]=\sup _{\theta \in \Theta} E_{\theta}[\cdot]$. In most cases, this set is often treated as an uncertain model of probabilities $\left\{P_{\theta}: \theta \in \Theta\right\}$ and the notion of sublinear expectation provides a robust way to measure a risk loss $X$. In fact, nonlinear expectation theory provides many rich, flexible, and elegant tools and plays an important role in many aspects. In particular, its important application in stochastic dominance, stochastic differential game, financial mathematics, economics, and partial differential equations attracted a large number of mathematicians, economists, and financial experts to join the research, for instance, the application of nonlinear expectation in the dynamic measurement and dominance of financial risk, backward stochastic differential equation theory and its application in financial products innovation, pricing, and so forth. We can see its recent developments from the following literature [7-12].

In this paper, we are interested in

$$
\bar{E}[\cdot]=\sup _{P \in \mathscr{P}} E_{P}[\cdot],
$$

where $\mathscr{P}$ is a set of probability measures, especially set $\bar{V}(A)=\bar{E}\left[I_{A}\right]=\sup _{P \in \mathscr{P}} E_{P}\left[I_{A}\right], \forall A \in \mathscr{F}$. Obviously, $\bar{V}$ is a capacity. Under the sublinear expectation, the upper bound of Cramér's theorem has come to a conclusion similar to the linear expectation (see [13]). On this basis, additionally, the main aim of this paper is to obtain Gärtner-Ellis's upper bound for the capacity $\bar{V}$.

This paper is organized as follows. In Section 2, we give some notions and lemmas that are useful in this paper. In Section 3, we give the main result including the proof. In 
Section 4, we give a brief application of our result in the classical risk model.

\section{Preliminaries}

We present some preliminaries in the theory of sublinear expectations. More details of this section can be found in Peng $[6,14,15]$.

Definition 1. Let $\Omega$ be a given set and let $\mathscr{H}$ be a linear space of real valued functions defined on $\Omega$. We assume that all constants are in $\mathscr{H}$ and that $X \in \mathscr{H}$ implies $|X| \in \mathscr{H}$. $\mathscr{H}$ is considered as the space of our "random variables." A nonlinear expectation $\widehat{E}$ on $\mathscr{H}$ is a functional $\widehat{E}: \mathscr{H} \mapsto \mathbb{R}$ satisfying the following properties: for all $X, Y \in \mathscr{H}$, one has

(a) monotonicity: if $X \geqslant Y$, then $\widehat{E}[X] \geqslant \widehat{E}[Y]$;

(b) constant preserving: $\widehat{E}[c]=c, c \in \mathbb{R}$.

The triple $(\Omega, \mathscr{H}, \widehat{E})$ is called a nonlinear expectation space (compare with a probability space $(\Omega, \mathscr{H}, P)$ ). We are mainly concerned with sublinear expectation where the expectation $\widehat{E}$ satisfies also

(c) subadditivity: $\widehat{E}[X]-\widehat{E}[Y] \leqslant \widehat{E}[X-Y]$;

(d) positive homogeneity: $\widehat{E}[\lambda X]=\lambda \widehat{E}[X], \forall \lambda \geqslant 0$.

If only (c) and (d) are satisfied, $\widehat{E}$ is called a sublinear functional.

The following representation theorem for sublinear expectations is very useful (see Peng $[6,15]$ for the proof).

Lemma 2. Let $\widehat{E}$ be a sublinear functional defined on $(\Omega, \mathscr{H})$; that is, (c) and (d) hold for $\widehat{E}$. Then there exists a family $\left\{E_{\theta}\right.$ : $\theta \in \Theta\}$ of linear functionals on $(\Omega, \mathscr{H})$ such that

$$
\widehat{E}[X]=\max _{\theta \in \Theta} E_{\theta}[X] .
$$

If (a) and (b) also hold, then $E_{\theta}$ are linear expectations for $\theta \epsilon$ $\Theta$. If we make, furthermore, the following assumption.

(H1) For each sequence $\left\{X_{n}\right\}_{n=1}^{\infty} \subset \mathscr{H}$ such that $X_{n}(\omega) \downarrow 0$ for $\omega$, one has $\widehat{E}\left[X_{n}\right] \downarrow 0$.

Then for each $\theta \in \Theta$, there exists a unique ( $\sigma$-additive) probability measure $P_{\theta}$ defined on $(\Omega, \sigma(\mathscr{H}))$ such that

$$
E_{\theta}[X]=\int_{\Omega} X(\omega) d P_{\theta}(\omega), \quad X \in \mathscr{H} .
$$

In this paper, we are interested in the following sublinear expectation:

$$
\bar{E}[\cdot]=\sup _{P \in \mathscr{P}} E_{P}[\cdot]
$$

where $\mathscr{P}$ is a set of probability measures. Let $\Omega$ be a given set and let $\mathscr{F}$ be a $\sigma$-algebra. Define $\bar{V}(A):=\bar{E}\left[I_{A}\right]=$ $\sup _{P \in \mathscr{P}} E_{P}\left[I_{A}\right], \forall A \in \mathscr{F}$; then $\bar{V}$ is a capacity.
Let $C\left(\mathbb{R}^{n}\right)$ denote the space of continuous functions defined on $\mathbb{R}^{n}$.

Now we recall some important notions of sublinear expectations distributions (see Peng $[6,14,15]$ ).

Definition 3. Let $X_{1}$ and $X_{2}$ be two random variables in a sublinear expectation space $(\Omega, \mathscr{F}, \bar{E})$. They are called identically distributed, denoted by $X_{1} \sim X_{2}$, if for $\varphi \in C(\mathbb{R})$, $\bar{E}\left[\varphi\left(X_{1}\right)\right]$ and $\bar{E}\left[\varphi\left(X_{2}\right)\right]$ exist; then one has

$$
\bar{E}\left[\varphi\left(X_{1}\right)\right]=\bar{E}\left[\varphi\left(X_{2}\right)\right] .
$$

Definition 4. In a sublinear expectation space $(\Omega, \mathscr{F}, \bar{E})$, a random vector $Y=\left(Y_{1}, \ldots, Y_{n}\right)$ is said to be independent of another random vector $X=\left(X_{1}, \ldots, X_{m}\right)$, if for $\varphi \in$ $C\left(\mathbb{R}^{m+n}\right), \bar{E}[\varphi(X, Y)]$ and $\bar{E}\left[\bar{E}[\varphi(x, Y)]_{x=X}\right]$ exist; then one has

$$
\bar{E}[\varphi(X, Y)]=\bar{E}\left[\bar{E}[\varphi(x, Y)]_{x=X}\right]
$$

We conclude this section with some notations on large deviations under a sublinear expectation [16].

Let $S$ be a topology space and $\delta$ be a $\sigma$-algebra on $S$. Let $\left(U_{n}, n \geq 1\right)$ be a family of measurable maps from $\Omega$ into $S$ and $b(n), n \geq 1$ be a positive function satisfying $b(n) \rightarrow \infty$ as $n \rightarrow \infty$. A nonnegative function $I$ on $S$ is called a (good) rate function if $\{I \leq l\}$ is (compact) closed for all $0 \leq l<\infty$.

$\left(\bar{V}\left(U_{n} \in \cdot\right), n \geq 1\right)$ is said to satisfy large deviation principle (LDP) with speed $b(n)$ and with rate function $I(x)$ if for any measurable closed subset $F \subset S$,

$$
\limsup _{n \rightarrow \infty} \frac{1}{b(n)} \log \bar{V}\left(U_{n} \in F\right) \leq-\inf _{x \in F} I(x)
$$

and for any measurable open subset $O \subset S$,

$$
\liminf _{n \rightarrow \infty} \frac{1}{b(n)} \log \bar{V}\left(U_{n} \in O\right) \geq-\inf _{x \in O} I(x) .
$$

Equations (7) and (8) are referred, respectively, to as upper bound of large deviations (ULD) and lower bound of large deviations (LLD).

$\left(\bar{V}\left(U_{n} \in \cdot\right), n \geq 1\right)$ is said to be exponentially tight if for any $L>0$, there exists a compact set $K_{L} \subset S$ such that

$$
\limsup _{n \rightarrow \infty} \frac{1}{b(n)} \log \bar{V}\left(U_{n} \in K_{L}^{c}\right) \leq-L .
$$

$\left(\bar{V}\left(U_{n} \in \cdot\right), n \geq 1\right)$ is said to satisfy $w$-upper bound of large deviations with speed $b(n)$ and with rate function $I(x)$ if (7) for any compact subset $F \subset S$.

It is known that if $\left(\bar{V}\left(U_{n} \in \cdot\right), n \geq 1\right)$ satisfies $w$-large deviation principle with speed $b(n)$ and with rate function $I$ and is exponentially tight, then it satisfies large deviation principle with speed $b(n)$ and with rate function $I$.

Definition 5. For any rate function $I$ and any $\delta>0$, the $\delta$-rate function is defined as

$$
I^{\delta}(x) \triangleq \min \left\{I(x)-\delta, \frac{1}{\delta}\right\}
$$


While in general $I^{\delta}$ is not a rate function, its usefulness stems from the fact that for any set $\Gamma$,

$$
\lim _{\delta \rightarrow 0} \inf _{x \in \Gamma} I^{\delta}(x)=\inf _{x \in \Gamma} I(x) .
$$

Consequently, the upper bound in (7) is equivalent to the statement that for any $\delta>0$ and for any measurable set $\Gamma$,

$$
\limsup _{n \rightarrow \infty} \frac{1}{b(n)} \log \bar{V}\left(U_{n} \in \Gamma\right) \leq-\inf _{x \in \bar{\Gamma}} I^{\delta}(x) .
$$

\section{Main Result}

In this section, firstly let us present some notations and assumptions that are used in the following details.

Consider a sequence of random vectors $X_{n} \in \mathbb{R}^{d}$; let $\left\{X_{n} ; n \geq 1\right\}$ be identically distributed under $\bar{E}[\cdot]$, where $X_{n}$ possesses logarithmic moment generating function $\bar{\Lambda}_{n}(\lambda):=$ $\log \bar{E}\left[e^{\left\langle\lambda, X_{n}\right\rangle}\right], \forall \lambda \in \mathbb{R}^{d}$. We also assume that each $X_{n+1}$ is independent of $\left(X_{1}, \ldots, X_{n}\right)$ for $n=1,2, \ldots$ under $\bar{E}[\cdot]$. Denote $\bar{S}_{n}=(1 / n) \sum_{i=1}^{n} X_{i}$.

Specifically, the following assumption is imposed throughout this section.

Assumption 6. For each $\lambda \in \mathbb{R}^{d}$, the logarithmic moment generating function, defined as the limit

$$
\bar{\Lambda}(\lambda):=\lim _{n \rightarrow \infty} \frac{1}{n} \bar{\Lambda}_{n}(n \lambda)
$$

exists as an extended real number. Furthermore, the origin belongs to the interior of $\mathscr{D}_{\bar{\Lambda}}:=\{\lambda: \bar{\Lambda}(\lambda)<\infty\}$.

Define

$$
\begin{gathered}
x:=-\bar{E}[-X] ; \\
\overline{\Lambda^{*}}(x):=\sup _{\lambda \in R}[\lambda x-\bar{\Lambda}(\lambda)], \quad \forall x \in \mathbb{R}^{d},
\end{gathered}
$$

where $\overline{\Lambda^{*}}(\cdot)$ is the Fenchel-Legendre transform of $\bar{\Lambda}(\cdot)$, with $\mathscr{D}_{\overline{\Lambda^{*}}}:=\left\{\lambda: \overline{\Lambda^{*}}(\lambda)<\infty\right\}$.

We always assume that

(H2) if $A_{n} \uparrow \Omega$, then $\bar{V}\left(A_{n}\right) \uparrow 1$.

Lemma 7. Let $N$ be a fixed integer. Then, for every $a_{\varepsilon}^{i} \geq 0$,

$$
\limsup _{\varepsilon \rightarrow 0} \varepsilon \log \left(\sum_{i=1}^{N} a_{\varepsilon}^{i}\right)=\max _{i=1}^{N} \limsup _{\varepsilon \rightarrow 0} \varepsilon \log a_{\varepsilon}^{i}
$$

Proof. First note that for all $\varepsilon$,

$$
0 \leq \varepsilon \log \left(\sum_{i=1}^{N} a_{\varepsilon}^{i}\right)-\max _{i=1}^{N} \varepsilon \log a_{\varepsilon}^{i} \leq \varepsilon \log N .
$$

Since $N$ is fixed, $\varepsilon \log N \rightarrow 0$ as $\varepsilon \rightarrow 0$ and

$$
\limsup _{\varepsilon \rightarrow 0} \max _{i=1}^{N} \varepsilon \log a_{\varepsilon}^{i}=\max _{i=1}^{N} \limsup \varepsilon \log a_{\varepsilon}^{i} .
$$

The next lemma describes a property of $\overline{\Lambda^{*}}(\cdot)$, which will be used to give a more accurate expression of the rate function.

Lemma 8. If $\log \bar{E} e^{\lambda X_{1}}<+\infty$ for some $\lambda \in \mathbb{R}^{+}$, then for any $x \geq \underline{x}, \overline{\Lambda^{*}}(x)=\sup _{\lambda \geq 0}\left\{\lambda x-\log \bar{E} e^{\lambda X_{1}}\right\}$ and for any $x \leq \underline{x}$, $\overline{\Lambda^{*}}(x)=\sup _{\lambda \leq 0}\left\{\lambda x-\log \bar{E} e^{\lambda X_{1}}\right\}$.

Here, we omit the proof of Lemma 8 (refer to [13] or [17]). The following theorem is the main result of this paper.

Theorem 9. Let Assumption 6 hold. Then we have for any closed set $F \subset \mathbb{R}^{d}$,

$$
\limsup _{n \rightarrow \infty} \frac{1}{n} \log \bar{V}\left(\bar{S}_{n} \in F\right) \leq-\inf _{x \in F} \overline{\Lambda^{*}}(x),
$$

where $\overline{\Lambda^{*}}(\cdot)$ is a convex rate function.

Proof. As mentioned in Section 2, establishing the upper bound is equivalent to proving that for every $\delta>0$ and every closed set $F \subset \mathbb{R}^{d}$,

$$
\limsup _{n \rightarrow \infty} \frac{1}{n} \log \bar{V}\left(\bar{S}_{n} \in F\right) \leq \delta-\inf _{x \in F} I^{\delta}(x),
$$

where $I^{\delta}$ is the $\delta$-rate function associated with $\overline{\Lambda^{*}}$. Fix a compact set $\Gamma \subset \mathbb{R}^{d}$. For every $q \in \Gamma$, choose $\lambda_{q} \in \mathbb{R}^{d}$ such that

$$
\left\langle\lambda_{q}, q\right\rangle-\bar{\Lambda}\left(\lambda_{q}\right) \geq I^{\delta}(q) .
$$

This is feasible on account of the definitions of $\overline{\Lambda^{*}}$ and $I^{\delta}$. For each $q$, choose $\rho_{q}>0$ such that $\rho_{q}\left|\lambda_{q}\right| \leq \delta$ and let $B_{q, \rho_{q}}=$ $\left\{x:|x-q|<\rho_{q}\right\}$ be the ball with center at $q$ and radius $\rho_{q}$. Observe for every $n, \lambda \in \mathbb{R}^{d}$, and measurable $G \subset \mathbb{R}^{d}$ that

$$
\begin{aligned}
\bar{V}\left(\bar{S}_{n} \in G\right) & =\bar{E}\left[1_{\bar{S}_{n} \in G}\right] \\
& \leq \bar{E}\left[\exp \left(n\left\langle\lambda, \bar{S}_{n}\right\rangle-\inf _{x \in G}\{n\langle\lambda, x\rangle\}\right)\right] .
\end{aligned}
$$

In particular, for each $n$ and $q \in \Gamma$,

$$
\begin{aligned}
\bar{V}\left(\bar{S}_{n}\right. & \left.\in B_{q, \rho_{q}}\right) \\
& \leq \bar{E}\left[\exp \left(n\left\langle\lambda_{q}, \bar{S}_{n}\right\rangle\right)\right] \exp \left(-\inf _{x \in B_{q, p_{q}}}\left\{n\left\langle\lambda_{q}, x\right\rangle\right\}\right) .
\end{aligned}
$$

Also, for any $q \in \Gamma$,

$$
-\inf _{x \in B_{q, \rho_{q}}}\left\langle\lambda_{q}, x\right\rangle \leq \rho_{q}\left|\lambda_{q}\right|-\left\langle\lambda_{q}, q\right\rangle \leq \delta-\left\langle\lambda_{q}, q\right\rangle,
$$

and therefore,

$$
\begin{aligned}
\frac{1}{n} \log \bar{V}\left(\bar{S}_{n} \in B_{q, \rho_{q}}\right) & \leq-\inf _{x \in B_{q, p_{q}}}\left\langle\lambda_{q}, x\right\rangle+\bar{\Lambda}\left(\lambda_{q}\right) \\
& \leq \delta-\left\langle\lambda_{q}, q\right\rangle+\bar{\Lambda}\left(\lambda_{q}\right) .
\end{aligned}
$$


Since $\Gamma$ is compact, one may extract from the open covering $\bigcup_{q \in \Gamma} B_{q, \rho_{q}}$ of $\Gamma$ a finite covering that consists of $N=N(\Gamma, \delta)<$ $\infty$ such balls with centers $q_{1}, \ldots, q_{N}$ in $\Gamma$. By the union of events bound and the preceding inequality,

$$
\begin{aligned}
\frac{1}{n} \log \bar{V}\left(\bar{S}_{n} \in \Gamma\right) \leq & \frac{1}{n} \log N+\delta \\
& -\min _{i=1, \ldots, N}\left\{\left\langle\lambda_{q_{i}}, q_{i}\right\rangle-\bar{\Lambda}\left(\lambda_{q_{i}}\right)\right\} .
\end{aligned}
$$

Hence, by our choice of $\lambda_{q}$,

$$
\limsup _{n \rightarrow \infty} \frac{1}{n} \log \bar{V}\left(\bar{S}_{n} \in \Gamma\right) \leq \delta-\min _{i=1, \ldots, N} I^{\delta}\left(q_{i}\right) .
$$

Since $q_{i} \in \Gamma$, the upper bound (7) is established for all compact sets.

As described earlier in Section 2, the upper bound of large deviations is extended to all closed subsets of $\mathbb{R}^{d}$ by showing that $\bar{V}\left(\bar{S}_{n} \in \cdot\right)$ is an exponentially tight family of probability measures. Let $H_{\rho} \triangleq[-\rho, \rho]^{d}$. Since $H_{\rho}^{c}=\bigcup_{j=1}^{d}\left\{x:\left|x^{j}\right|>\rho\right\}$, the union of events bound yields

$$
\begin{aligned}
\bar{V}\left(\bar{S}_{n} \in H_{\rho}^{c}\right) \leq & \sum_{j=1}^{d} \bar{V}\left(\bar{S}_{n}^{j} \in[\rho, \infty)\right) \\
& +\sum_{j=1}^{d} \bar{V}\left(\bar{S}_{n}^{j} \in(-\infty,-\rho]\right),
\end{aligned}
$$

where $\bar{S}_{n}^{j}, j=1, \ldots, d$ are the coordinates of the random vector $\bar{S}_{n}$; namely, $\left(\bar{V}\left(\bar{S}_{n}^{j} \in \cdot\right)\right)$ are the laws governing $(1 / n)$ $\sum_{i=1}^{n} X_{i}^{j}$. Let $\mathbf{e}_{j}$ denote the $j$ th unit vector in $\mathbb{R}^{d}$ for $j=$ $1, \ldots, d$. Since $0 \in \mathscr{D}_{\frac{1}{\Lambda}}^{0}$ (refer to [13, Lemma 3.1]), there exist $\theta_{j}>0$ and $\eta_{j}>0$ such that $\bar{\Lambda}\left(\theta_{j} \mathbf{e}_{j}\right)<\infty$ and $\bar{\Lambda}\left(-\eta_{j} \mathbf{e}_{j}\right)<\infty$ for $j=1, \ldots, d$. By Chebyshev's inequality, we have

$$
\begin{array}{r}
\bar{V}\left(\bar{S}_{n}^{j} \in(-\infty,-\rho]\right) \leq \exp \left(-n \eta_{j} \rho+\bar{\Lambda}_{n}\left(-n \eta_{j} \mathbf{e}_{j}\right)\right), \\
\bar{V}\left(\bar{S}_{n}^{j} \in[\rho, \infty)\right) \leq \exp \left(-n \theta_{j} \rho+\bar{\Lambda}_{n}\left(n \theta_{j} \mathbf{e}_{j}\right)\right), \\
j=1, \ldots, d .
\end{array}
$$

Hence, for $j=1, \ldots, d$,

$$
\begin{gathered}
\lim _{\rho \rightarrow \infty} \limsup _{n \rightarrow \infty} \frac{1}{n} \log \bar{V}\left(\bar{S}_{n}^{j} \in(-\infty,-\rho]\right)=-\infty, \\
\lim _{\rho \rightarrow \infty} \limsup _{n \rightarrow \infty} \frac{1}{n} \log \bar{V}\left(\bar{S}_{n}^{j} \in[\rho, \infty)\right)=-\infty .
\end{gathered}
$$
Lemma 7,

Consequently, by the union of events bound and

$$
\lim _{\rho \rightarrow \infty} \limsup _{n \rightarrow \infty} \frac{1}{n} \log \bar{V}\left(\bar{S}_{n} \in H_{\rho}^{c}\right)=-\infty .
$$

Therefore, $\bar{V}\left(\bar{S}_{n} \in \cdot\right)$ is an exponentially tight family of probability measures, since the hypercubes $H_{\rho}$ are compact.
Remark 10. Since $\bar{E}$ is not linear, Cramér's method is not useful for lower bound of large deviations. This is consistent with the conclusion of [13]. In the paper [13], the author gives a counter example to illustrate that under the sublinear expectation, the lower bound of Cramér's theorem is not obtained. Since Gärtner-Ellis theorem be a generalization of Cramér's theorem in non-i.i.d situation to conclusions, we see under the assumptions of theorem, the lower bound of Gärtner-Ellis theorem does not hold.

\section{Application}

In this section, we consider the classical risk process under sublinear expectation $\bar{E}[\cdot]$. The classical risk process $\left(R^{x}(t)\right)_{t \geq 0}$ is defined by

$$
R^{x}(t)=x+p t-S(t), \quad t \geq 0,
$$

where $x>0$ is the initial capital and $p>0$ is the (constant) premium rate, and the aggregate claims process $(S(t))_{t \geq 0}$ is a compound Poisson process. More precisely we have $S(t)=$ $\sum_{i=1}^{N(t)} X_{i}$ and $N(t)=\sum_{i \geq 1} 1_{\left\{T_{i} \leq t\right\}}$, where $\left\{X_{i}\right\}_{i \geq 1}$ is a sequence of positive random variables, $(N(t))_{t \geq 0}$ is a counting process with points $\left\{T_{i}\right\}_{i \geq 1},\left\{X_{i}\right\}_{i \geq 1}$, and $(N(t))_{t \geq 0}$ independent, the $X_{i}, i=1,2, \ldots$, are independent and identically distributed, and where $(N(t))_{t \geq 0}$ is a Poisson process with intensity $\mu$ under linear expectation. Now we consider $(N(t))_{t \geq 0}$ is a $G$ Poisson process (its definition refers to [18]) under sublinear expectation $\bar{E}[\cdot]$. Then, $(S(t))_{t \geq 0}$ is a compound $G$-Poisson process correspondingly.

We also assume the following superexponential condition holds for the random variables $\left\{X_{i}\right\}_{i \geq 1}$ under sublinear expectation $\bar{E}[\cdot]$.

Assumption 11. $\bar{E}\left[e^{\lambda X_{1}}\right]<\infty$ for all $\lambda \in \mathbb{R}$.

Let $\varphi_{N(t)}(\lambda)$ be the moment generating function (m.g.f.) of $(N(t))_{t \geq 0}$; that is,

$$
\varphi_{N(t)}(\lambda)=\bar{E} e^{\lambda N(t)}, \quad t \geq 0, \forall \lambda \in \mathbb{R} .
$$

Then its logarithmic moment generating function is expressed as follows:

$$
\Lambda_{N(t)}(\lambda) \triangleq \log \bar{E} e^{\lambda N(t)}=\log \varphi_{N(t)}(\lambda) .
$$

Let $\psi_{N(t)}(\lambda)$ be the limit of the normalized logarithmic moment generating function of $(N(t))_{t \geq 0}$; that is,

$$
\psi_{N(t)}(\lambda)=\lim _{t \rightarrow \infty} \frac{1}{t} \log \bar{E} e^{\lambda N(t)} .
$$

In order to obtain the m.g.f. of the process $(S(t))_{t \geq 0}$, firstly we introduce a lemma which plays a role in the next lemma. We omit its proof which can be found in [19, Lemma 1.1].

Lemma 12. If a sequence of $d$-dimensions random variables $\left\{X_{i}, i=1, \ldots, m\right\}$ under sublinear expectation space $(\Omega, \mathscr{H}, \bar{E})$ satisfies for any $i=1, \ldots, m-1, X_{i+1}$ is independent of $\left(X_{1}, \ldots, X_{i}\right)$, then the following conclusions are established. 
(1) If $\varphi_{1}, \ldots, \varphi_{m}$ are lower semicontinuous functions in $\mathbb{R}^{d} \rightarrow[0,+\infty)$, one has

$\bar{E}\left(\varphi_{1}\left(X_{1}\right) \cdots \varphi_{m}\left(X_{m}\right)\right)=\bar{E}\left(\varphi_{1}\left(X_{1}\right)\right) \cdots \bar{E}\left(\varphi_{m}\left(X_{m}\right)\right)$.

(2) If $\psi_{1}, \ldots, \psi_{m}$ are upper semicontinuous functions in $\mathbb{R}^{d} \rightarrow[0,+\infty)$ and there exists a continuous function $\Psi$ such that $\Psi\left(X_{i}\right) \in \mathbb{L}_{c}^{1} \triangleq\left\{X \in \mathbb{L}^{1} ; X\right.$ is quasicontinuous and $\left.\lim _{n \rightarrow \infty} \bar{E}\left[|X| I_{|X|>n}\right]=0\right\}$, and $\psi_{i} \leq \Psi$, for any $i=1, \ldots, m$, one has

$$
\bar{E}\left(\psi_{1}\left(X_{1}\right) \cdots \psi_{m}\left(X_{m}\right)\right)=\bar{E}\left(\psi_{1}\left(X_{1}\right)\right) \cdots \bar{E}\left(\psi_{m}\left(X_{m}\right)\right) .
$$

Lemma 13. If $(N(t))_{t \geq 0}$ and $\left\{X_{i}\right\}_{i \geq 1}$ are independent, then for each $\lambda \in \mathbb{R}$

$$
\varphi_{S(t)}(\lambda)=\varphi_{N(t)}\left(\Lambda_{X_{1}}(\lambda)\right), \quad t \geq 0
$$

Proof. By Lemma 12, we have

$$
\begin{aligned}
\varphi_{S(t)}(\lambda) & =\bar{E}\left[e^{\lambda S(t)}\right]=\bar{E}\left[e^{\lambda \sum_{i=1}^{N(t)} X_{i}}\right] \\
& =\bar{E}\left[\bar{E}\left[e^{\lambda \sum_{i=1}^{k} X_{i}}\right]_{k=N(t)}\right]=\bar{E}\left[\left[\bar{E}\left[e^{\lambda X_{1}}\right]\right]_{k=N(t)}^{k}\right] \\
& =\bar{E}\left[\left(\bar{E} e^{\lambda X_{1}}\right)^{N(t)}\right]=\bar{E}\left[e^{N(t) \log \bar{E}\left[e^{\lambda X_{1}}\right]}\right] \\
& =\varphi_{N(t)}\left(\Lambda_{X_{1}}(\lambda)\right), \quad t \geq 0 .
\end{aligned}
$$

This completes the proof.

Then we can see

$$
\begin{aligned}
\lim _{t \rightarrow \infty} \frac{1}{t} \log \bar{E}\left[e^{\lambda S(t)}\right] & =\lim _{t \rightarrow \infty} \frac{1}{t} \log \varphi_{N(t)}\left(\Lambda_{X_{1}}(\lambda)\right) \\
& =\psi_{N(t)}\left(\Lambda_{X_{1}}(\lambda)\right) .
\end{aligned}
$$

That is to say, the normalized logarithmic moment generating function of $(S(t))_{t \geq 0}$ has a limit. By Theorem 9 that we obtained in Section 3, we can say $(S(t))_{t \geq 0}$ satisfies the upper bound of Gärtner-Ellis theorem with rate function $I$ defined by

$$
I(x)=\sup _{\lambda \in \mathbb{R}}\left\{\lambda x-\psi_{N(t)}\left(\Lambda_{X_{1}}(\lambda)\right)\right\}, \quad \forall x \in \mathbb{R} .
$$

Next, we give you a brief description about G-Poisson process in Ren's Ph.D. thesis [17]. Let $(N(t))_{t \geq 0}$ be G-Poisson process under sublinear expectation $\bar{E}[\cdot]$. Then, $\bar{E}[\varphi(x+$ $N(t))$ ] satisfies the following one-dimensional equation:

$$
\begin{array}{r}
\partial_{t} u(t, x)-G(u(t, x+1)-u(t, x))=0, \\
u(0, x)=\varphi(x), \quad 0 \leq t \leq 1,
\end{array}
$$

where $G(a)=\mu_{2} a^{+}-\mu_{1} a^{-}, 0 \leq \mu_{1} \leq \mu_{2}$. Referring to [18], we know, for any increasing function $\phi$,

$$
\bar{E}[\phi(x+N(t))]=\sum_{i=0}^{\infty} \phi(x+i) \frac{\left(\mu_{2} t\right)^{i}}{i !} e^{-\mu_{2} t},
$$

and for any decreasing function $\phi$,

$$
\bar{E}[\phi(x+N(t))]=\sum_{i=0}^{\infty} \phi(x+i) \frac{\left(\mu_{1} t\right)^{i}}{i !} e^{-\mu_{1} t} .
$$

Then we have $\log \bar{E} e^{\lambda N(t)}=\mu_{2} t\left(e^{\lambda}-1\right)$, for any $\lambda \geq 0$, and $\log \bar{E} e^{\lambda N(t)}=\mu_{1} t\left(e^{\lambda}-1\right)$, for any $\lambda \leq 0$.

Since $X_{i}$ represents the amount claimed, we know $X_{i} \geq 0$ and $i \geq 1$; and so $\Lambda_{X_{1}}(\lambda)=\log \bar{E}\left[e^{\lambda X_{1}}\right] \geq 0$. In the above formula (42), we set $x=0$,

$$
\begin{aligned}
\varphi_{N(t)}\left(\Lambda_{X_{1}}(\lambda)\right) & =\bar{E}\left[e^{N(t) \Lambda_{X_{1}}(\lambda)}\right] \\
& =\sum_{i=0}^{\infty} e^{i \Lambda_{X_{1}}(\lambda)} \frac{\left(\mu_{2} t\right)^{i}}{i !} e^{-\mu_{2} t} \\
& =\sum_{i=0}^{\infty} \frac{\left(\mu_{2} t e^{\Lambda_{X_{1}}(\lambda)}\right)^{i}}{i !} e^{-\mu_{2} t} \\
& =e^{\mu_{2} t\left(e^{\Lambda_{X_{1}}(\lambda)}-1\right)} .
\end{aligned}
$$

Substituting this result into (39), we have

$$
\lim _{t \rightarrow \infty} \frac{1}{t} \log \bar{E}\left[e^{\lambda S(t)}\right]=\mu_{2}\left(e^{\Lambda_{X_{1}}(\lambda)}-1\right) .
$$

By (40) and Lemma 8, we get the rate function of $(S(t))_{t \geq 0}$ in the upper bound of Gärtner-Ellis theorem. As described below, for $x \geq \underline{x}$,

$$
\begin{aligned}
I(x) & =\sup _{\lambda \in \mathbb{R}}\left\{\lambda x-\psi_{N(t)}\left(\Lambda_{X_{1}}(\lambda)\right)\right\} \\
& =\sup _{\lambda \geq 0}\left\{\lambda x-\mu_{2}\left(e^{\Lambda_{X_{1}}(\lambda)}-1\right)\right\} .
\end{aligned}
$$

The above result can be calculated further, specifically according to the different distributions of the amount claimed.

\section{Conflict of Interests}

The authors declare that there is no conflict of interests regarding the publication of this paper.

\section{References}

[1] A. Dembo and O. Zeitouni, Large Deviations Techniques and Applications, Springer, New York, NY, USA, 2nd edition, 1998.

[2] S. Peng, "G-Expectation, G-Brownian motion and related stochastic calculus of Itô's type," in Proceedings of the 2005 Abel Symposium, vol. 2, pp. 541-567, Springer, 2006.

[3] P. Artzner, F. Delbaen, J.-M. Eber, and D. Heath, "Coherent measures of risk," Mathematical Finance, vol. 9, no. 3, pp. 203228, 1999. 
[4] H. Föllmer and A. Schied, "Convex measures of risk and trading constraints," Finance and Stochastics, vol. 6, no. 4, pp. 429-447, 2002.

[5] M. Frittelli and E. Rossaza Gianin, "Dynamic convex risk measures," in New Riak Measures for the 21st Century, G. Szegö, Ed., pp. 227-248, John Wiley \& Sons, New York, NY, USA, 2004.

[6] S. Peng, "Survey on normal distributions, central limit theorem, Brownian motion and the related stochastic calculus under sublinear expectations," Science in China A: Mathematics, vol. 52, no. 7, pp. 1391-1411, 2009.

[7] L. G. Epstein and S. Ji, "Ambiguous volatility, possibility and utility in continuous time," Journal of Mathematical Economics, vol. 50, pp. 269-282, 2014.

[8] L. G. Epstein and S. Ji, "Ambiguous volatility and asset pricing in continuous time," Review of Financial Studies, vol. 26, no. 7, pp. 1740-1786, 2013.

[9] M. Hu, S. Ji, S. Peng, and Y. Song, "Backward stochastic differential equations driven by G-Brownian motion," Stochastic Processes and Their Applications, vol. 124, no. 1, pp. 759-784, 2014.

[10] M. Hu, S. Ji, S. Peng, and Y. Song, "Comparison theorem, Feynman-Kac formula and Girsanov transformation for BSDEs driven by G-Brownian motion," Stochastic Processes and Their Applications, vol. 124, no. 2, pp. 1170-1195, 2014.

[11] M. Hu, S. Ji, and S. Yang, "A stochastic recursive optimal control problem under the G-expectation framework," Applied Mathematics \& Optimization, 2014.

[12] X. Xiao, "Stochastic dominance under the nonlinear expected utilities," . In press.

[13] F. Hu, "On Cramér's theorem for capacities," Comptes Rendus de l'Académie des Sciences I, vol. 348, no. 17-18, pp. 1009-1013, 2010.

[14] S. Peng, "G-Brownian motion and dynamic risk measure under volatility uncertainty," http://arxiv.org/abs/0711.2834.

[15] S. Peng, "A new central limit theorem under sublinear expectations," http://arxiv.org/abs/0803.2656.

[16] F. Gao and H. Jiang, "Large deviations for stochastic differential equations driven by G-Brownian motion," Stochastic Processes and Their Applications, vol. 120, no. 11, pp. 2212-2240, 2010.

[17] L. Ren, G-Lévy processes in finite and infinite dimensional space and related topics [Ph.D. thesis], 2012.

[18] M. Hu and S. Peng, "G-Lévy processesunder sublinear expectations," http://arxiv.org/abs/0911.3533.

[19] F. Gao and M. Xu, "Large deviations and Moderate deviations of independent random variables under sublinear expectations," Science China Mathematics, vol. 41, no. 4, pp. 337-352, 2011 (Chinese). 


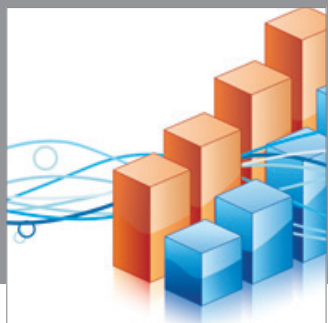

Advances in

Operations Research

mansans

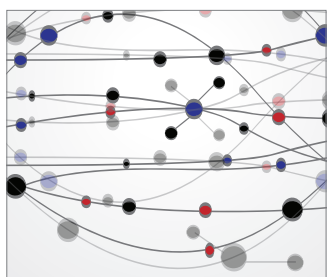

The Scientific World Journal
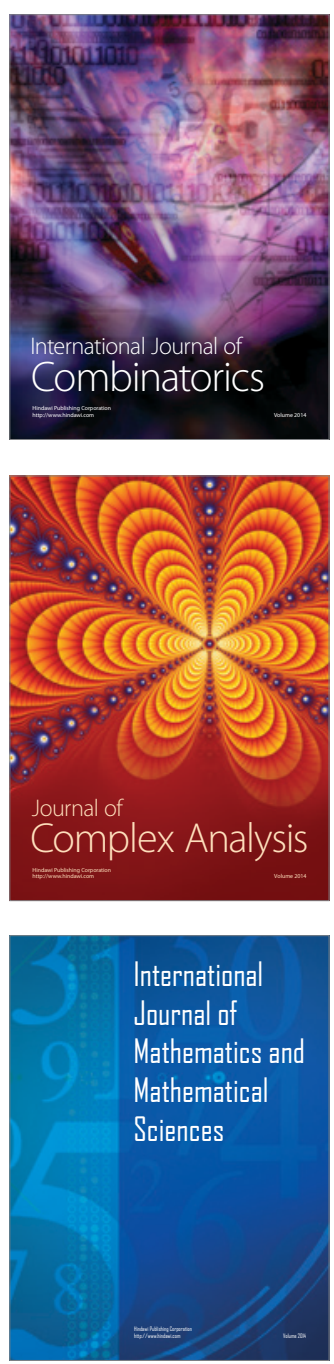
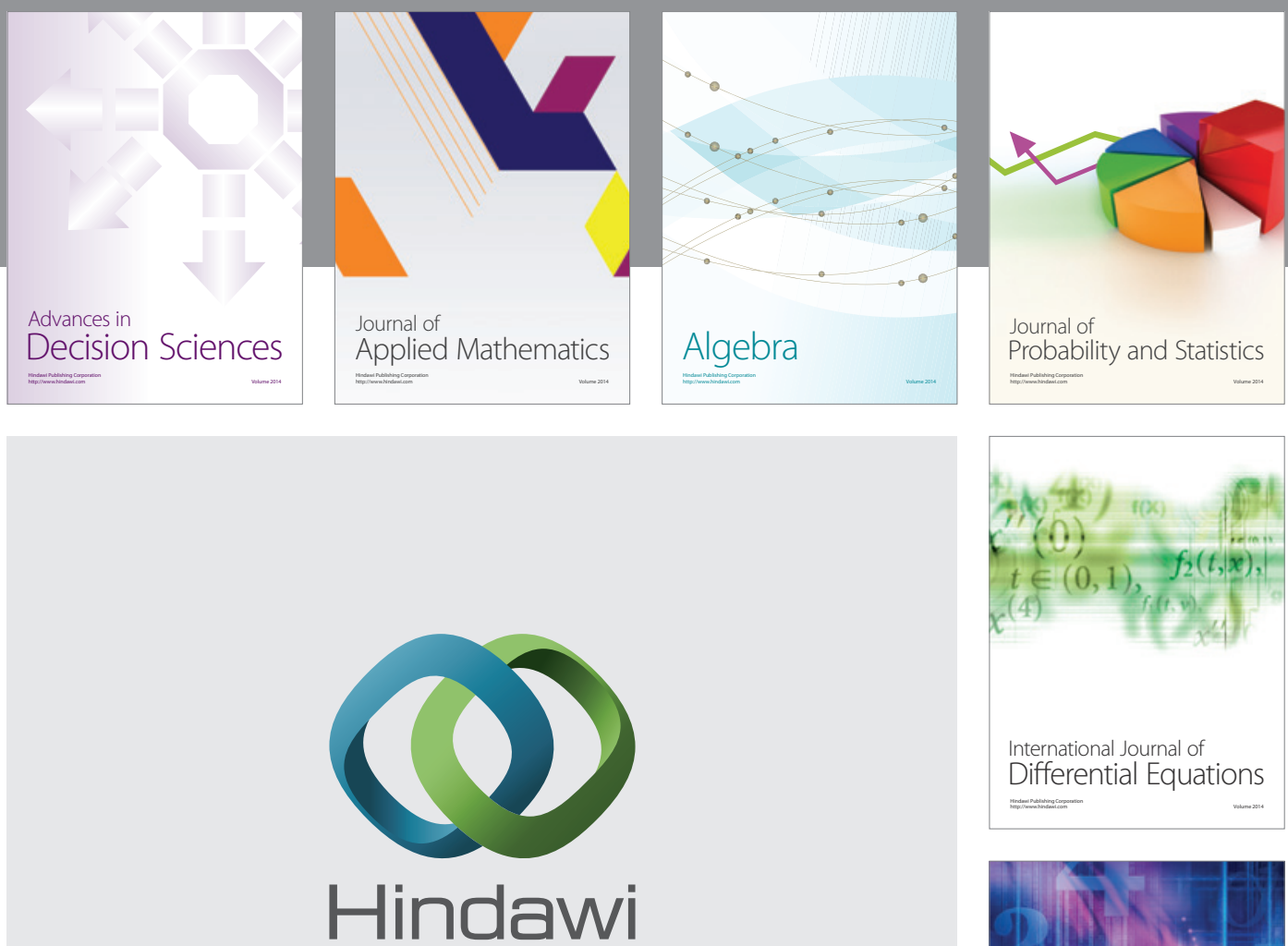

Submit your manuscripts at http://www.hindawi.com
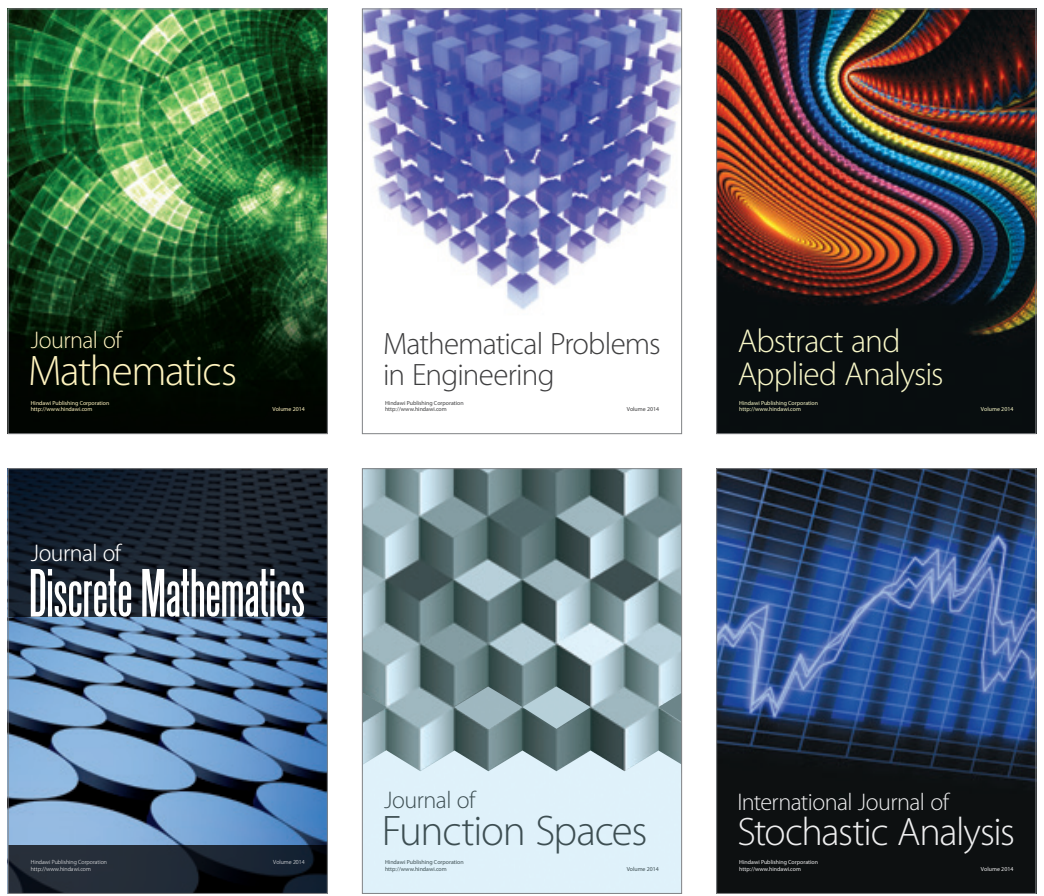

Journal of

Function Spaces

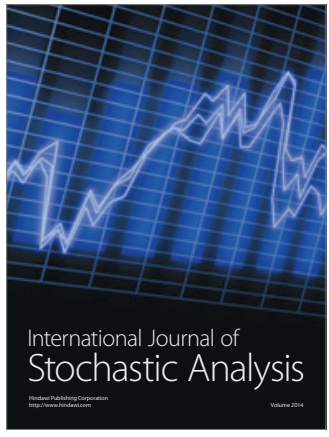

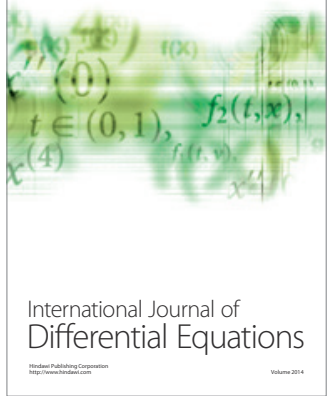
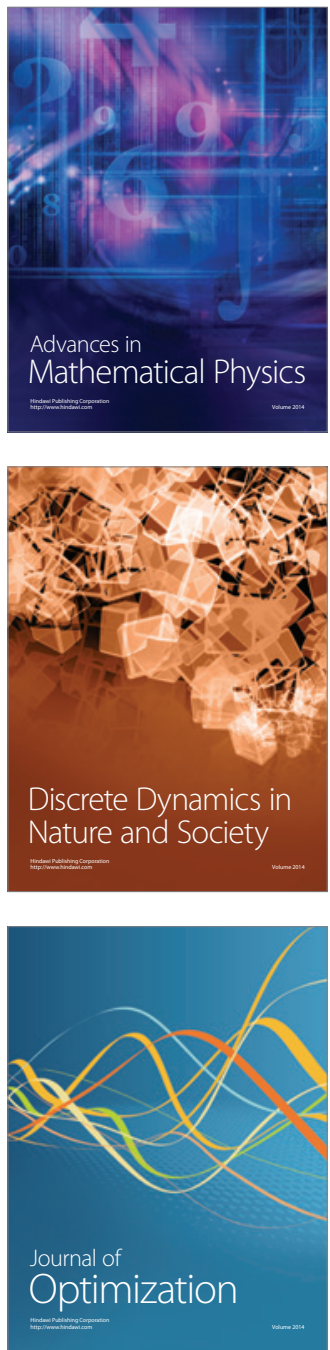